\title{
COMPUTER BASED LEARNING MODALITIES IN ANATOMY DISSECTION
}

\author{
B. I. Navin Kumar ${ }^{1}$, Krupa Sailaja Tadepalli ${ }^{* 2}$, G. R. Raveena Paul ${ }^{3}$, T. K. Rajasree ${ }^{4}$, \\ Sayeeda Anjum ${ }^{5}$.
}

${ }^{1}$ Associate Professor, Department of Anatomy, Malla Reddy Institute of Medical Sciences, Suraram, Hyderabad, Telangana, India.

${ }^{* 2}$ Associate Professor, Department Of Anatomy, Maheshwara Medical College \& Hospital, Chitkul (V), Near Isnapur X Roads, Patancheru, Telangana, India.

3,5 Assistant Professor, Department of Anatomy, Malla Reddy Institute of Medical Sciences, Suraram, Hyderabad, Telangana, India.

${ }^{4}$ Professor, Department of Anatomy, Malla Reddy Institute of Medical Sciences, Suraram, Hyderabad, Telangana, India.

\section{ABSTRACT}

Background: Cadaveric dissection has been used for centuries for teaching gross anatomy all over the world. It has been considered as a necessity to learn gross anatomy and helps the medical students in understanding the three-dimensional relationship of different anatomical structures and variations. But the paucity of cadavers and high financial cost has considerably contributed to the development of alternative teaching techniques. Advancement in web-based medical technology leads to the development of virtual dissection programs. These programs have been found to be an effective way to teach anatomy and are being preferred over cadaveric dissection. Is cadaveric dissection simply a rite of passage or is it a necessity? The aim of our study was to determine whether cadaveric dissection is a necessity in medical education, and if it can be replaced by newer alternative techniques like computer based dissection procedure.

Materials and Methods: A batch of 150, $1^{\text {st }}$ MBBS students of Malla Reddy Institute of Medical Sciences, Hyderabad were studied by dividing them into 2 groups of 75 each after subjecting them to Conventional based Learning (CNVL) of dissection versus Computer Based Learning (CBL). A questionnaire was given to both the students and the faculty. Based on the response to the questionnaire statistical analysis was done.

Results: The present study indicated that the Computer Based Learning in dissection (CBL) method was found to be more effective method for teaching anatomy by $79 \%$ of the students and $84 \%$ of faculty doctors. The value $r=0.89$ shows the pre \& post score has correlated positively and has an effect of study. (Paired Two Sample t-test: $p=3.24 E-29<0.05)$. As well the results were found to be increased with the pre $(35 \%)$ to post test $(65 \%)$ and $p<0.05$ implies the effect.

Conclusion: The three dimensional (3D) modeling of anatomical structures in the human body was presented in detailed step by step cadaveric dissections. It also provided detailed human anatomical training for students, where there is a lack of cadaver facility and where there are more students to cadaver ratio. Thus, the multimedia equipped interactive anatomical laboratory software enhance both memorization and visual learning skills of the medical students.

KEYWORDS: Cadaveric Dissection, Computer based learning modalities.

Address for Correspondence: Dr. Krupa SailajaTadepalli, Associate Professor, Department Of Anatomy, Maheshwara Medical College \& Hospital, Chitkul (V), Near Isnapur X Roads,Patancheru, Telangana. 502307, India. Mobile No: 9703924748 E-Mail:drsailajatk@gmail.com

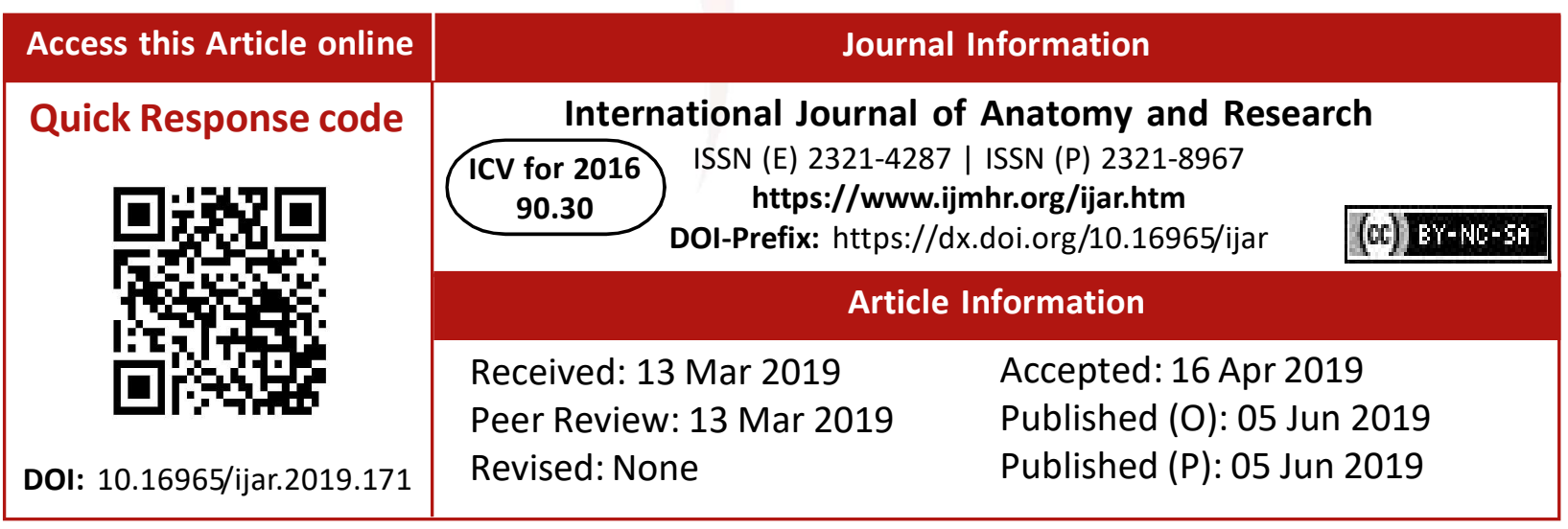




\section{INTRODUCTION}

Anatomy is derived from the Greek word "ana= body, tome= cutting" which means cutting up. Cadaveric dissection has been the paradigm of anatomy teaching since the Renaissance, and defining experience of medical teaching since the 16th and 17 th centuries [1]. It has become a greatly acknowledged fact that good medical or surgical practice could only be based on adequate anatomical knowledge which can only be learned from cadaveric dissection [2].

Medical education's paradigm has shifted towards problem-based learning and integrated curriculum. This has resulted in the studying of core basic science subjects including anatomy in the light of clinical context only and abandoning cadaveric dissection. Alternative teaching methods are now more commonly deployed in teaching gross anatomy. This issue will become more common with the passage of time. The age-old debate 'to dissect, or not to dissect' still rages on [2].

There is an ongoing debate about how to teach anatomy in student-centered, problem-based learning (PBL) context. Literature suggests the existence of two perspectives i.e., the traditionalists who favor teaching through dissection of human cadaversand reformers who advocate for the use of a variety of modalities, including prosected specimens, plastinated specimens, computer-assisted software and radiological imaging, with cadaveric dissection included as just another strategy to support student's learning in anatomy [3].

\section{MATERIALS AND METHODS}

150 Students of First M.B.B.S of Malla Reddy Institute of Medical Sciences were involved in the present study. A self-constructed questionnaire was used to assess the perception of students and faculty about cadaveric dissection and other alternative anatomy teaching methods. Students were subjected to a pre-test evaluation on the concerned topic of dissection and were divided into 2 groups. One group was included in Conventional Based Learning [CNVL] and other in Computer Based Learning [CBL]. After which the groups were interchanged and evaluated with post-test evaluation. The faculty perceptions were also taken into consideration during the presentstudy. This study was done for a period of 4 months i.e., from October 2017 to February 2018. SPSS version 21 was used to analyze the data. Chi-square test was calculated.

\section{RESULTS}

\section{Format of questionnaire}

\begin{tabular}{|l|}
\hline SQ: STUDENT QUESTIONNAIRE \\
\hline 1. Easy to learn \\
\hline 2. Less time for learning \\
\hline 3. Practical knowledge of the student \\
\hline 4. In-depth learning \\
\hline 5. Interesting \\
\hline 6. Group learning \\
\hline 7. Real time effectiveness \\
\hline
\end{tabular}

\begin{tabular}{|l|}
\hline FQ: Faculty Questionnaire \\
\hline 1. Easy method to teach \\
\hline 2. Comfortable for group teaching \\
\hline 3. Cost \& Time effective \\
\hline 4. Real time effectiveness \\
\hline 5. Faculty preference \\
\hline 6. Limited resources available \\
\hline
\end{tabular}

Scores: Strongly Agree $(S A)=5$,

Agree $(A)=2$

\section{Disagree $(D A)=0$}

Fig. 1: Average score of Student Opinion on the basis of Questionnaire (SQs).

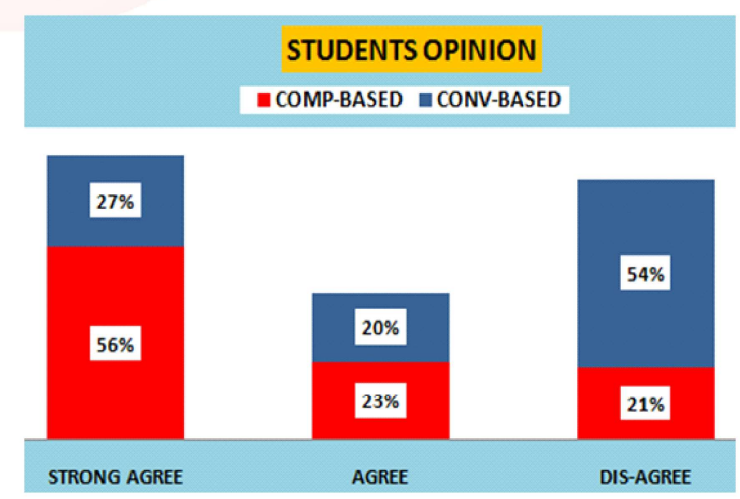

Fig. 2: Average score of Faculty Opinion on the basis of Questionnaire (FQs).

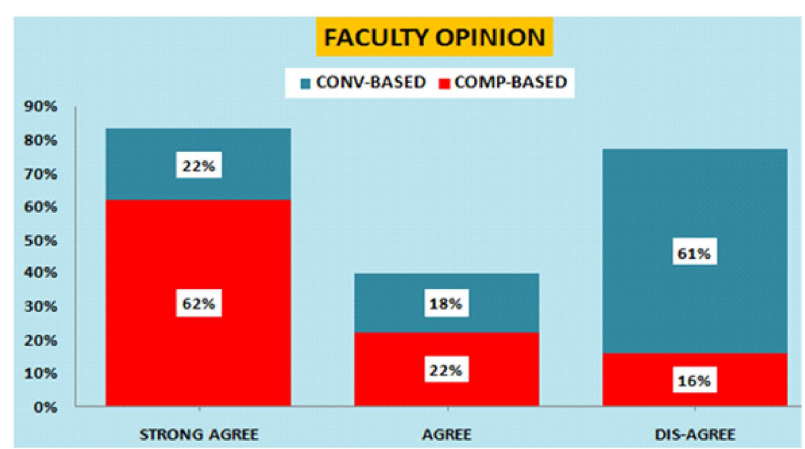


Fig. 3\&4: Comparison of Pre \&Post test.

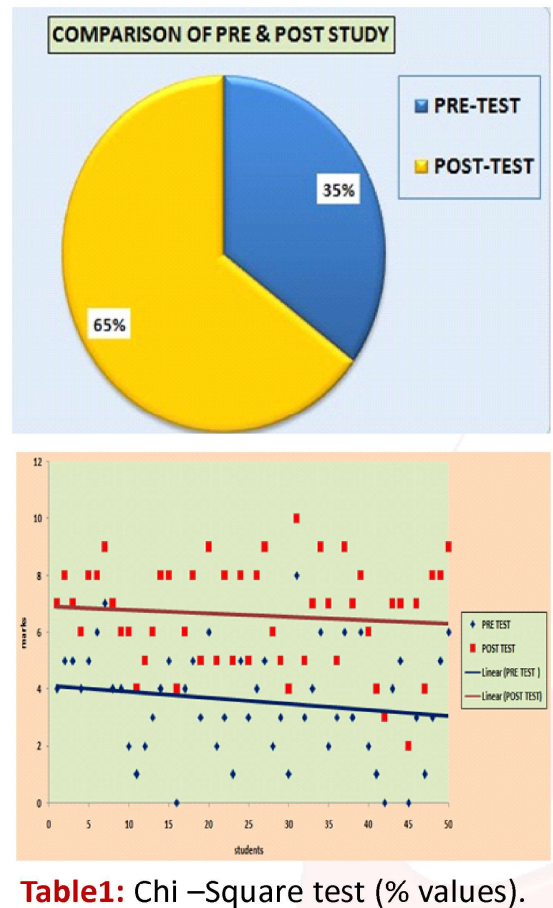

STUDENT OPINION $\quad$ FACULTYOPINION

\begin{tabular}{|c|r|r|r|r|r|r|r|}
\hline \multicolumn{4}{|c|}{ STUDENT OPINION } & \multicolumn{4}{c|}{ FACUITY OPINION } \\
\hline AGREE(\%) & COMP & CNVL & TOTAL & AGREE (\%) & COMP & CNVL & TOTAL \\
\hline YES(SA+A) & 79 & 46 & 125 & YES (SA+A) & 84 & 39 & 123 \\
\hline NO (DA) & 21 & 54 & 75 & NO (DA) & 16 & 61 & 77 \\
\hline TOTAL & 100 & 100 & 200 & TOTAL & 100 & 100 & 200 \\
\hline p-value & \multicolumn{3}{|c|}{0.000003} & & p-value & \multicolumn{3}{|c}{$<0.0000001$} \\
\hline
\end{tabular}

In the present study the Computer Based Learning (CBL) method was found to be more effective and preferable by both the students (79\%) \&the faculty (84\%). This was shown using a Bar Diagram and pie diagrams in Figure 1 and 2. This was statistically correlated using chi-square test. The value $r=0.89$ shows the pre \& post score is correlated positively and has an effect of study. (Paired Two Sample t-test: $p=3$. $24 \mathrm{E}-29<0.05)$.

The results were found to be increased from pre $(35 \%)$ to post test $(65 \%)$ and $p<0.05$ implies the effect.(Fig-3\&4).

\section{DISCUSSION}

Anatomy and dissection have long been considered a milestone of medical education. During the Renaissance, Anatomists began to dissect in order to investigate the structure of the body and produced texts illustrated with images based on their dissections. The era of scientific human anatomy is highlighted by the publication of the main opera from Andreas Vesalius (von Wesel), the real Father of modern anatomy.
Towards the end of 20th century, dissection was the core basis in medical education, and adequate anatomical knowledge is essential for surgeons and those who perform invasive procedures on patients. Anatomical knowledge is also pivotal to complete a medical examination, to make a diagnosis and also to properly communicate with colleagues [4].

Today, Africa and the USA are the leading geographic areas in which medical schools offers Cadaveric Dissection(CD). In Africa, 90\% of medical schools and also in North and South America, a large majority of medical schools offer $C D$ in their undergraduate curricula. $A$ survey involving 65 medical schools in the USA indicated the inclusion of dissection in most anatomy teaching programs. In Canada, several medical schools have retained mandatory dissection in their undergraduate medical curriculum; the University of Saskatchewan offers optional dissection to interested students. In Asia, data regarding CD is not available from most of the medical schools. However, the literature shows that, in India, Sri Lanka, Bangladesh, China, Korea and Thailand many medical schools continue teaching anatomy assisted by $\mathrm{CD}[5]$.

According to Tam MDBS, advantages for the use of dissection include conceptualization of the multi-dimensional nature of the human body, touch-mediated perception, and appreciation of anatomical variability, the acquisition of practical dissection skills and appreciation of the ethical and moral issues arising from contact with cadavers [6].

According to Bekele[7] et al. (2011), several medical schools in the United Kingdom, for example, have phased out dissection altogether. But, active dissection programs still remain in the United States, Germany and Africa. According to studies the reduction in anatomical teaching has consequences, and, this is being experienced in regard to patient care.

Since the inception of the modern medical curriculum, anatomy teaching and cadaver dissection (CD) has been diluted or reallocated in medical training. Anatomists, surgeons, and medical educationists have published different opinions on the status of $\mathrm{CD}[5]$. 
B. I. Navin Kumar, Krupa Sailaja Tadepalli, et al., COMPUTER BASED LEARNING MODALITIES IN ANATOMY DISSECTION.

Over a period of time, arousal of many issues such as establishment of many new private medical institutions of various specialized disciplines have overgrown the need for cadavers has also increased proportionately, where bodies for dissection are scarce. As Cadavers are embalmed with a fluid containing glycerin, ethyl alcohol and phenol, physical contact of one's skin and clothing with the cadaver is becoming a major health issue.

In our study, students with access to dissection rated it to be less useful in comparison to computer-based dissection. The reasons could be inappropriate student cadaver ratio (50:1), conditions of poor ventilation and pungent smell in the dissection halls, and varying pace of learning between every student. There is continuing debate concerning the best way to teach anatomy. The Peninsula Medical School, UK, is teaching anatomy without cadavers. They argue that doctors encounter anatomy in clinical practice through living anatomy and medical imaging and therefore seem to believe that it makes sense to teach anatomy in these contexts right from the beginning [6].

Computer assisted learning (CAL) enables undergraduate institution which lack cadavers, the opportunity to offer a detailed course in human anatomy through stimulated dissection of system or region. It offers comparison of the structures with radiographic and magnetic resonance images in addition to the histological appearance of that structure. It allows a structure to be zoomed and rotation of the images between the four views (i.e., anterior, posterior, medial, lateral). The operator can also specify a structure by name and can obtain a highlighted view, or ask for an identifying label on the structure, by projecting images on to a large screen for lecture purposes. Computer software program has the facility to use pathological material as an illustrative point where regional anatomy is being demonstratedand, through this there is an option of incorporating existing teaching material into study guides, sample question, histological and radiographic material[8].

In our study most of the students and the faculty has mentioned several attributes for computer assisted learning modalities in
Anatomy dissection with the advantage that the learning could be adapted to the need of the individual, and can be used at the student's pace which is always available. This has the greatest potential with interactive computer resources using virtual dissections, animations or decision making. Electronic resources have the potential to replicate learning based on the curiosity and exploration, which are the fundamentals to adult learning.

Alternatively CAL can be used as a supplement to the current teaching methods, deliver material and is particularly useful in demonstrating topics which are complex or relatively difficult to access when seen in the textbook or cadaver[6].

\section{CONCLUSION}

In conclusion, this article reported on the value of dissection for the First year medical students. Positive perceptions and experiences were reported by majority of the students for computer based learning in dissection rather than conventional dissection. Few medical students offered suggestions which would help to solidify their anatomical knowledge. Finally, this study confirmed that Computer Assisted Learning modalities in Anatomy dissection provided the best anatomical knowledge.

However, dissection is still considered as the best by several doctors as it forms a valuable source of learning anatomy. The future of teaching anatomy does not depend on any single method. It is, in fact, usingthe right combination of all available resources in an interactive way that maximizes positive outcomes.

\section{Conflicts of Interests: None}

\section{REFERENCES}

[1]. Shaikh ST. Cadaver Dissection in Anatomy: The Ethical Aspect. AnatPhysiol. 2015;\$5:007.

[2]. Haider Ghazanfar, Sannah Rashid, AshrafHussain, Madiha Ghazanfar, Ali Ghazanfar, Arshad Javaid. Cadaveric Dissection a Thing of the Past? The Insight of Consultants, Fellows, and Residents Cureus. 2018 Apr;10(4):e2418.

[3]. Van Wyk, J. \&Rennie, C.O. Learning Anatomy Through Dissection:Perceptions of a Diverse Medical Student CohortInt. J. Morphol., 2015;33(1):89-95.

[4]. Veronica Papa, Mauro Vaccarezza.Teaching Anatomy in the XXI Century: New Aspects and Pitfalls.ScientificWorldJournal. 2013;310-348. 
[5]. Ismail Memon.Cadaver Dissection Is Obsolete in Medical Training! A Misinterpreted Notion.Med PrincPract. 2018 Jul;27(3):201-210.

[6]. Tam MDB, Hart AR, Williams S, Heylings D \& Leinster S. Is learning anatomy facilitated by computer-aided learning? A review of the literature. 2009;31:e393e396.

[7]. Bekele A,Reissig, D. Löffler S, Hinz A. Experiences with dissection courses in human anatomy: a comparison between Germany and Ethiopia. Ann. Anat., 2011;193(2):163-7.
[8]. Fazal-Ur-Rehman, SheebaNuzhat Khan, S. Mobashir Yunus. Students, perception of computer assisted teaching and learning of anatomy-in a scenario where cadavers are lacking. Biomedical Research 2012;23(2):215-218.

How to cite this article:

B. I. Navin Kumar, Krupa Sailaja Tadepalli, G. R. Raveena Paul, T. K. Rajasree, Sayeeda Anjum. COMPUTER BASED LEARNING MODALITIES IN ANATOMY DISSECTION. Int J Anat Res 2019;7(2.3):6607-6611. DOI: 10.16965/ijar.2019.171 